\title{
The influence of surgical technique on early posttransplant atrial fibrillation - comparison of biatrial, bicaval, and total orthotopic heart transplantation
}

\author{
This article was published in the following Dove Press journal: \\ Therapeutics and Clinical Risk Management \\ 8 March 2017 \\ Number of times this article has been viewed
}

\author{
Rasmus Rivinius' \\ Matthias Helmschrott ${ }^{\prime}$ \\ Arjang Ruhparwar ${ }^{2}$ \\ Christian Erbel' \\ Christian A Gleissner' \\ Fabrice F Darche' \\ Dierk Thomas' \\ Tom Bruckner ${ }^{3}$ \\ Hugo A Katus' \\ Andreas $\mathrm{O}$ Doesch $^{1}$ \\ 'Department of Cardiology, \\ Angiology and Pneumology, \\ ${ }^{2}$ Department of Cardiac Surgery, \\ Heidelberg University Hospital, \\ ${ }^{3}$ Institute for Medical Biometry and \\ Informatics, University of Heidelberg, \\ Heidelberg, Germany
}

Purpose: Early posttransplant atrial fibrillation (AF) has been associated with worse clinical outcomes after heart transplantation (HTX). The type of surgical technique may constitute a relevant risk factor for AF.

Patients and methods: This retrospective single-center study included 530 adult patients. Patients were stratified by surgical technique (biatrial, bicaval, or total orthotopic HTX) and early posttransplant heart rhythm ( $\mathrm{AF}$ or sinus rhythm). Univariate and multivariate analyses were performed to evaluate risk factors for AF.

Results: A total of 161 patients received biatrial HTX (30.4\%), 115 bicaval HTX (21.7\%), and 254 total orthotopic HTX (47.9\%). Sixty-one of 530 patients developed early posttransplant AF (11.5\%). Patients with AF showed a statistically inferior 5-year survival compared to those with sinus rhythm $(P<0.0001)$. Total orthotopic HTX had the lowest rate of AF (total orthotopic HTX [6.3\%], bicaval HTX [14.8\%], biatrial HTX [17.4\%], $P=0.0012)$. Multivariate analysis showed pretransplant valvular heart disease $(P=0.0372)$, posttransplant enlarged left atrium ( $P=0.0066)$, posttransplant mitral regurgitation $(P=0.0370)$, and non-total orthotopic HTX $(P=0.0112)$ as risk factors for AF.

Conclusion: Early posttransplant AF was associated with increased mortality $(P<0.0001)$. Total orthotopic HTX showed the lowest rate of AF compared to biatrial or bicaval $\operatorname{HTX}(P=0.0012)$.

Keywords: atrial fibrillation, biatrial heart transplantation, bicaval heart transplantation, mortality, surgical technique, total orthotopic heart transplantation

\section{Introduction}

Atrial fibrillation (AF) in the early posttransplant period is a common phenomenon in patients after heart transplantation (HTX) with a previously described incidence of $7.9 \%-18.2 \% .^{1,2}$ Although several risk factors, such as immunosuppressive drug therapy, acute rejection, or ischemic time, may have an influence on the occurrence of AF, the type of surgical technique could play a key role in the development of posttransplant AF within 30 days after HTX. ${ }^{1-7}$

A number of different cardiac transplantation techniques currently exist. In clinical routine, three types are commonly used: biatrial (BA), bicaval (BC), and total orthotopic (TO) HTX. The initial "standard technique" for HTX, also known as "biatrial technique (BA)", was developed by Lower and Shumway. ${ }^{3}$ It consists of two long anastomoses of donor and recipient atria resulting in enlarged cavities with distorted
Correspondence: Andreas O Doesch Department of Cardiology, Angiology and Pneumology, Heidelberg University Hospital, Im Neuenheimer Feld 4I0, 69120 Heidelberg, Germany

Tel +49622I 5639936

$\mathrm{Fax}+496221564105$

Email andreas.doesch@med.uniheidelberg.de 
anatomy, long suture lines with potentially proarrhythmic scar tissue, and possible sinus node injury. ${ }^{4,5}$

An alternative is the "bicaval technique (BC)", also known as "bicaval, left atrial technique", clinically introduced by Sievers et al. ${ }^{6}$ Here, the right donor atrium is preserved via donor-to-recipient venae cavae anastomoses resulting in unaltered properties of the right atrial geometry and conduction system. ${ }^{4,5}$ However, the left atrial anastomosis is still performed via donor-to-recipient atrial anastomosis as described by Lower and Shumway. ${ }^{3,4}$

"Total orthotopic (TO) HTX", also known as "bicaval, bipulmonary venous technique", was clinically introduced by Dreyfus et al. ${ }^{7}$ This technique comprises the total excision of the recipient atria, except for two small pulmonary vein cuffs, which are separately integrated into the left donor atrium..$^{4,8,9}$ The right donor atrium is connected via $\mathrm{BC}$ anastomosis as reported by Sievers et al. ${ }^{6}$ In this way, the anatomic integrity and physiology of both atria are preserved. ${ }^{9}$ Disadvantages of this technique are prolonged ischemic time as a result of complex anastomoses and pulmonary venous stenosis. ${ }^{4}$

Therefore, the aim of this study was to determine whether the type of surgical technique (BA, BC, or TO) has an influence on early posttransplant AF.

\section{Patients and methods}

\section{Patients}

This study was performed in accordance with the ethical standards of the Declaration of Helsinki and was approved by the Ethics Committee of the University of Heidelberg, Heidelberg, Germany. It included all patients ( $\geq 18$ years) receiving HTX at the Heidelberg Heart Center, Heidelberg, Germany, between June 1989 and December 2012. Patients with repeated HTX were excluded. Data were retrieved from the clinical routine. Written informed consent was obtained for the Heidelberg HTX register. ${ }^{10,11}$ As only clinical routine data were used for this study, no additional written informed consent was required from the patients.

Patients were stratified by surgical technique (BA, BC, or TO) and according to posttransplant heart rhythm within 30 days after HTX. The decision of which surgical technique to be used had not been preselected. Factors influencing the choice of HTX technique were surgeon's preference, anatomical characteristics, and previous open heart surgery.

\section{Follow-up}

Patients were routinely followed-up according to the center standard. The minimum follow-up period was 30 days after HTX. During the early posttransplant period, heart rhythm was continuously assessed by monitor telemetry. Furthermore, 12-lead electrocardiography was routinely performed and in case of arrhythmias on monitor telemetry. Additionally, 24-hour-Holter recording was performed. All available source files pertaining to heart rhythm in the early posttransplant period ( $\leq 30$ days after HTX) were analyzed. Early posttransplant AF (AF $\leq 30$ days after HTX) was defined as AF lasting 30 seconds or longer. Atrial flutter or other supraventricular tachyarrhythmias were not included.

Cardiac rejection episodes were diagnosed and treated according to the revised International Society for Heart and Lung Transplantation classification. ${ }^{12}$

\section{Posttransplant medication}

Patients after HTX received an immunosuppressive induction treatment with anti-thymocyte globulin since 1994. The initial immunosuppressive regimen of cyclosporine A (CsA) and azathioprine (AZA) was subsequently replaced by CsA and mycophenolate mofetil (MMF) from 2001 onward, and by tacrolimus (TAC) and MMF from 2006 onward. Simultaneously, steroids (prednisolone) were applied. ${ }^{10,11}$

\section{Statistical analysis}

Analysis of data was performed with SAS (version 9.3; SAS Institute, Cary, NC, USA). Data were expressed as mean \pm standard deviation (SD) or as absolute number (n) with percentage (\%). Student's $t$-test and analysis of variance were used for continuous variables, and a chi-square test was used for categorical variables. A Kaplan-Meier estimator was used to estimate the survival of patients after HTX. A $P$-value of $<0.0500$ was considered to be statistically significant. ${ }^{10,11}$

Univariate analyses covered demographic data, perioperative data, initial posttransplant medication, including immunosuppressive drug therapy, postoperative echocardiographic features, and treated rejection episodes (TREs) within 30 days after HTX.

Furthermore, a multivariate analysis (Cox regression model) was performed to estimate the influence of seven variables on AF within 30 days after HTX. It comprised five variables which were statistically significant risk factors for early posttransplant AF in the univariate analysis: valvular heart disease as principal diagnosis for HTX, donor age ( $\geq 40$ years), enlarged end-diastolic left atrial diameter after $\operatorname{HTX}(\geq 40 \mathrm{~mm})$, mitral regurgitation after HTX, and non-TO HTX (BA and BC). Additionally, two potential risk factors of clinical importance for AF were included: prolonged ischemic time ( $\geq 240$ minutes) and occurrence of a TRE within 30 days after HTX. No more variables were included to 
ensure a stable number of events (AF) per analyzed variable and to avoid biased regression coefficients.

\section{Results}

\section{Baseline characteristics by HTX technique}

Stratified by surgical technique, 161 patients received BA-HTX (30.4\%), 115 patients received BC-HTX (21.7\%), and 254 patients received TO-HTX (47.9\%). Comparison of the three techniques showed no statistically significant differences in age, male sex, coronary artery disease, arterial hypertension, dyslipidemia, or diabetes mellitus (all $P \geq 0.0500$ ). Patients with BA-HTX had a lower body mass index (BMI;

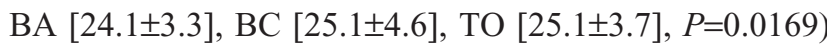
and a higher rate of renal insufficiency (BA [65.8\%], BC [55.7\%], TO [53.1\%], $P=0.0349)$. However, glomerular filtration rate (GFR) showed no statistically significant difference in the three groups ( $P=0.9078)$.

Comparison of previous open heart surgery of the recipient revealed no statistically significant differences in overall open heart surgery or in the two subgroups: coronary artery bypass graft surgery and congenital, valvular, or ventricular surgery (all $P \geq 0.0500$ ). More patients with BC-HTX and TO-HTX had prior ventricular assist device (VAD) surgery (BA [0.0\%], BC [3.5\%], TO [3.9\%], $P=0.0420$ ). However, only 14 of 530 patients $(2.6 \%)$ received a VAD before HTX.

In terms of principal diagnosis for HTX, there were no statistically significant differences between groups regarding nonischemic cardiomyopathy (CMP), ischemic CMP, or valvular heart disease (all $P \geq 0.0500$ ). The percentage of cardiac amyloidosis as principal diagnosis for HTX was higher in patients with BC-HTX and TO-HTX (BA [0.0\%], BC [11.3\%], TO [9.1\%], $P=0.0002$ ).

Considering donor data, donors of patients with BA-HTX were younger (BA [34.5 \pm 12.1 years], BC [42.8 \pm 13.3 years], TO [41.5 \pm 13.1 years], $P<0.0001$ ), more male sex (BA [62.7\%], BC [32.2\%], TO [39.0\%], $P<0.0001)$, and had a lower

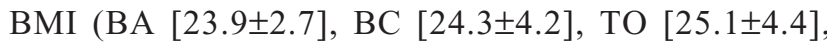
$P=0.0128)$.

Patients with BA-HTX had a lower rate of transplant sex mismatch (BA [31.1\%], BC [50.4\%], TO [47.6\%], $P=0.0008$ ), a shorter ischemic time (BA [168.3 \pm 58.1 minutes], BC [238.8 \pm 60.2 minutes], TO [226.3 \pm 58.2 minutes], $P<0.0001)$, and a higher percentage of occurrence of a TRE within 30 days (BA [36.6\%], BC [13.9\%], TO [11.0\%], $P<0.0001$ ) after HTX. The length of initial hospital stay was comparable between the three HTX techniques $(P=0.7361)$.
Patients with TO-HTX had the lowest rate of early posttransplant AF within 30 days after HTX (BA [17.4\%], BC [14.8\%], TO [6.3\%], $P=0.0012)$. Baseline characteristics by HTX technique are presented in Table 1.

\section{Baseline characteristics by heart rhythm}

Analysis of early posttransplant heart rhythm within 30 days after HTX showed 469 patients (88.5\%) with sinus rhythm (SR) and 61 patients (11.5\%) with AF.

There were no statistically significant differences between recipient data, previous open heart surgery of the recipient, or transplant sex mismatch in patients with or without AF (all $P \geq 0.0500$ ).

The principal diagnosis for HTX differed not significantly considering nonischemic CMP, ischemic CMP, or cardiac amyloidosis (all $P \geq 0.0500$ ). Patients with early posttransplant AF had a higher rate of valvular heart disease as principal diagnosis for HTX $(P=0.0284)$.

Regarding donor data, no statistically significant differences were found in donor sex $(P=0.2416)$ or donor BMI $(P=0.9680)$. Student's $t$-test using continuous variables showed a higher donor age in patients with AF (AF [43.9 \pm 13.1 years] vs SR [39.1 \pm 13.2 years], $P=0.0092)$, whereas a chi-square test using categorical variables showed no statistically significant difference between patients with or without AF considering donor age $\geq 40$ years (AF: 40 of 61 [65.6\%] vs SR: 255 of 469 [54.4\%], $P=0.0976$ ) or donor age $\geq 60$ years (AF: 5 of 61 [8.2\%] vs SR: 19 of 469 [4.1\%], $P=0.1430$ ).

There were no statistically significant differences between the two groups in ischemic time $(P=0.1618)$, the occurrence of a TRE within 30 days after $\operatorname{HTX}(P=0.7688)$, or the length of initial hospital stay $(P=0.8480)$.

Finally, patients with early posttransplant AF within 30 days after HTX received significantly more often BAHTX (AF: 28 of 61 [45.9\%] vs SR: 133 of 469 [28.4\%], $P=0.0051)$. In contrast, patients with SR within 30 days after HTX had significantly more often TO-HTX (AF: 16 of 61 [26.2\%] vs SR: 238 of 469 [50.7\%], $P=0.0003$ ).

No statistically significant difference between the two groups was found regarding BC-HTX (AF: 17 of 61 [27.9\%] vs SR: 98 of 469 [20.9\%], $P=0.2139)$. Baseline characteristics by heart rhythm are presented in Table 2 .

\section{Initial posttransplant medication By HTX technique}

Comparison of the three techniques indicated that patients with BA-HTX predominantly received CsA (BA [98.8\%], 
Table I Baseline characteristics by HTX technique

\begin{tabular}{|c|c|c|c|c|}
\hline Parameters & Biatrial $(n=161)$ & Bicaval $(n=I \mid 5)$ & Total orthotopic $(n=254)$ & $P$-value \\
\hline \multicolumn{5}{|l|}{ Recipient data } \\
\hline Age in years, mean $\pm S D$ & $51.2 \pm 10.3$ & $51.4 \pm 10.9$ & $52.3 \pm 10.4$ & 0.5514 \\
\hline Male sex, n (\%) & |3| (8I.4) & $83(72.2)$ & $198(78.0)$ & 0.1931 \\
\hline Body mass index, mean $\pm S D$ & $24.1 \pm 3.3$ & $25.1 \pm 4.6$ & $25.1 \pm 3.7$ & $0.0169 *$ \\
\hline Coronary artery disease, $\mathrm{n}(\%)$ & $67(4 I .6)$ & $49(42.6)$ & $106(4 I .7)$ & $0.984 I$ \\
\hline Arterial hypertension, n (\%) & $93(57.8)$ & $57(49.6)$ & $138(54.3)$ & 0.4030 \\
\hline Dyslipidemia, n (\%) & $99(61.5)$ & $74(64.3)$ & $166(65.4)$ & 0.7234 \\
\hline Diabetes mellitus, n (\%) & $63(39.1)$ & $34(29.6)$ & $85(33.5)$ & 0.2360 \\
\hline Renal insufficiency, ${ }^{\mathrm{a}} \mathrm{n}(\%)$ & $106(65.8)$ & $64(55.7)$ & $135(53.1)$ & $0.0349 *$ \\
\hline GFR, mean \pm SD & $60.3 \pm 15.3$ & $61.2 \pm 22.1$ & $60.2 \pm 23.3$ & 0.9078 \\
\hline \multicolumn{5}{|l|}{ Previous open heart surgery } \\
\hline Overall open heart surgery, n (\%) & $33(20.5)$ & $33(28.7)$ & $77(30.3)$ & 0.0804 \\
\hline CABG surgery, n (\%) & $16(9.9)$ & $14(12.2)$ & $42(16.5)$ & 0.1421 \\
\hline Congenital, valvular, or ventricular surgery, $\mathrm{n}(\%)$ & $18(11.2)$ & $16(13.9)$ & $29(11.4)$ & 0.7479 \\
\hline VAD surgery, n (\%) & $0(0.0)$ & $4(3.5)$ & $10(3.9)$ & $0.0420 *$ \\
\hline \multicolumn{5}{|l|}{ Principal diagnosis for HTX } \\
\hline Nonischemic CMP, n (\%) & $96(59.6)$ & $59(5 \mathrm{I} .3)$ & $129(50.8)$ & 0.1824 \\
\hline Ischemic CMP, n (\%) & $52(32.3)$ & $37(32.2)$ & $92(36.2)$ & 0.6283 \\
\hline Valvular heart disease, n (\%) & $13(8.1)$ & $6(5.2)$ & $10(3.9)$ & 0.1940 \\
\hline Cardiac amyloidosis, n (\%) & $0(0.0)$ & $13(11.3)$ & $23(9.1)$ & $0.0002^{*}$ \\
\hline \multicolumn{5}{|l|}{ Donor data } \\
\hline Age in years, mean $\pm S D$ & $34.5 \pm 12.1$ & $42.8 \pm 13.3$ & $41.5 \pm 13.1$ & $<0.0001 *$ \\
\hline Male sex, $\mathrm{n}(\%)$ & $101(62.7)$ & $37(32.2)$ & $99(39.0)$ & $<0.000 I^{*}$ \\
\hline Body mass index, mean $\pm S D$ & $23.9 \pm 2.7$ & $24.3 \pm 4.2$ & $25.1 \pm 4.4$ & $0.0128 *$ \\
\hline \multicolumn{5}{|l|}{ Transplant sex mismatch } \\
\hline Mismatch, n (\%) & $50(31.1)$ & $58(50.4)$ & $|2|(47.6)$ & $0.0008^{*}$ \\
\hline Donor $(\mathrm{m})$ to recipient $(\mathrm{f}), \mathrm{n}(\%)$ & $10(6.2)$ & $6(5.2)$ & II (4.3) & 0.6958 \\
\hline Donor $(\mathrm{f})$ to recipient $(\mathrm{m}), \mathrm{n}(\%)$ & $40(24.9)$ & $52(45.2)$ & $110(43.3)$ & $0.0002^{*}$ \\
\hline \multicolumn{5}{|l|}{ Perioperative data } \\
\hline Ischemic time (minutes), mean \pm SD & $168.3 \pm 58.1$ & $238.8 \pm 60.2$ & $226.3 \pm 58.2$ & $<0.000 I^{*}$ \\
\hline Ischemic time $\geq 240$ minutes, $n$ (\%) & $12(7.5)$ & $59(51.3)$ & $104(40.9)$ & $<0.000 I^{*}$ \\
\hline TREs $\leq 30$ days after $\mathrm{HTX}$, mean $\pm \mathrm{SD}$ & $0.5 \pm 0.8$ & $0.1 \pm 0.4$ & $0.1 \pm 0.4$ & $<0.000 I^{*}$ \\
\hline$\geq \mathrm{I}$ TRE $\leq 30$ days after HTX, $\mathrm{n}(\%)$ & $59(36.6)$ & $16(13.9)$ & $28(11.0)$ & $<0.000 I^{*}$ \\
\hline $\mathrm{AF} \leq 30$ days after $\mathrm{HTX}, \mathrm{n}(\%)$ & $28(17.4)$ & $17(14.8)$ & $16(6.3)$ & $0.0012^{*}$ \\
\hline Length of initial hospital stay (days), mean \pm SD & $42.2 \pm 20.1$ & $44.4 \pm 27.0$ & $42.8 \pm 22.0$ & $0.736 I$ \\
\hline
\end{tabular}

Notes: ${ }^{a} \mathrm{GFR}<60 \mathrm{~mL} / \mathrm{min} / 1.73 \mathrm{~m}^{2} ; * \mathrm{P}<0.05$, statistically significant.

Abbreviations: HTX, heart transplantation; SD, standard deviation; GFR, glomerular filtration rate; CABG, coronary artery bypass graft; VAD, ventricular assist device; CMP, cardiomyopathy; $m$, male; $f$, female; TRE, treated rejection episode; AF, atrial fibrillation; $n$, number.

BC [42.6\%], TO [52.0\%], $P<0.0001)$ and AZA (BA [98.8\%], BC [26.1\%], TO [30.7\%], $P<0.0001)$ as initial immunosuppressive drug therapy, while those with BCHTX and TO-HTX mainly received MMF (BA [1.2\%], BC [73.9\%], TO [69.3\%], $P<0.0001)$ concomitantly with CsA or TAC (BA [1.2\%], BC [57.4\%], TO [48.0\%], $P<0.0001$ ). Additionally, all patients were administered steroids concomitantly.

Patients with BC-HTX and TO-HTX received more often ivabradine $(P=0.0002)$ and calcium channel blocker $(P=0.0400)$. However, neither the administration of nondihydropyridine calcium channel blocker $(P=0.8937)$ nor the use of beta blocker $(P=0.4762)$ differed statistically significantly across the study.

\section{By heart rhythm}

There were no statistically significant differences between patients with SR or AF within 30 days after HTX regarding immunosuppressive drug therapy or antiarrhythmic medication (all $P \geq 0.0500$ ). Data for medication used within 30 days after HTX are given in Table 3.

\section{Echocardiographic features}

By HTX technique

More patients with BA-HTX $(95.7 \%, P<0.0001)$ had an enlarged right atrium (end-diastolic diameter $\geq 35 \mathrm{~mm}$ ) compared to those with BC-HTX (27.0\%) or TO-HTX (22.4\%). With regard to left atrial size, patients with TO-HTX (75.6\%, $P<0.0001$ ) had a higher percentage of a normal left atrial size 
Table 2 Baseline characteristics by heart rhythm within 30 days after HTX

\begin{tabular}{|c|c|c|c|}
\hline Parameters & Atrial fibrillation $(n=6 \mathrm{I})$ & Sinus rhythm $(n=469)$ & $P$-value \\
\hline \multicolumn{4}{|l|}{ Recipient data } \\
\hline Age in years, mean $\pm S D$ & $52.2 \pm 11.3$ & $51.7 \pm 10.3$ & 0.7434 \\
\hline Male sex, n (\%) & $45(73.8)$ & $367(78.3)$ & 0.4287 \\
\hline Body mass index, mean $\pm S D$ & $25 . I \pm 4.4$ & $24.8 \pm 3.7$ & 0.5933 \\
\hline Coronary artery disease, $\mathrm{n}(\%)$ & $28(45.9)$ & $194(4 I .4)$ & 0.4993 \\
\hline Arterial hypertension, $\mathrm{n}(\%)$ & $32(52.5)$ & $256(54.6)$ & 0.7539 \\
\hline Dyslipidemia, n (\%) & $38(62.3)$ & $301(64.2)$ & 0.7731 \\
\hline Diabetes mellitus, n (\%) & $25(41.0)$ & $157(33.5)$ & 0.2454 \\
\hline Renal insufficiency, ${ }^{a}$ ( (\%) & $36(59.0)$ & $269(57.4)$ & 0.8051 \\
\hline GFR, mean \pm SD & $59.7 \pm 19.4$ & $60.5 \pm 21.1$ & 0.7532 \\
\hline \multicolumn{4}{|l|}{ Previous open heart surgery } \\
\hline Overall open heart surgery, n (\%) & $19(31.1)$ & $124(26.4)$ & 0.4358 \\
\hline CABG surgery, $n(\%)$ & $9(14.8)$ & $63(13.4)$ & 0.7769 \\
\hline Congenital, valvular, or ventricular surgery, $\mathrm{n}(\%)$ & $10(16.4)$ & $53(11.3)$ & 0.2476 \\
\hline VAD surgery, $\mathrm{n}(\%)$ & I (I.7) & $13(2.8)$ & 0.6039 \\
\hline \multicolumn{4}{|l|}{ Principal diagnosis for HTX } \\
\hline Nonischemic CMP, n (\%) & $29(47.5)$ & $255(54.4)$ & 0.3143 \\
\hline Ischemic CMP, n (\%) & $22(36.1)$ & $159(33.9)$ & 0.7375 \\
\hline Valvular heart disease, $\mathrm{n}(\%)$ & $7(11.5)$ & $22(4.7)$ & $0.0284 *$ \\
\hline Cardiac amyloidosis, n (\%) & $3(4.9)$ & $33(7.0)$ & 0.5362 \\
\hline \multicolumn{4}{|l|}{ Donor data } \\
\hline Age in years, mean $\pm S D$ & $43.9 \pm 13.1$ & $39.1 \pm 13.2$ & $0.0092^{*}$ \\
\hline Male sex, $\mathrm{n}(\%)$ & $23(37.7)$ & $214(45.6)$ & 0.2416 \\
\hline Body mass index, mean $\pm S D$ & $24.5 \pm 3.3$ & $24.6 \pm 4.0$ & 0.9680 \\
\hline \multicolumn{4}{|l|}{ Transplant sex mismatch } \\
\hline Mismatch, n (\%) & $28(45.9)$ & $201(42.9)$ & 0.6516 \\
\hline Donor $(\mathrm{m})$ to recipient $(\mathrm{f}), \mathrm{n}(\%)$ & $3(4.9)$ & $24(5.1)$ & 0.9469 \\
\hline Donor (f) to recipient $(\mathrm{m}), \mathrm{n}(\%)$ & $25(41.0)$ & $177(37.8)$ & 0.6236 \\
\hline \multicolumn{4}{|l|}{ Perioperative data } \\
\hline Ischemic time (minutes), mean \pm SD & $223.2 \pm 70.0$ & $209.9 \pm 64.5$ & 0.1618 \\
\hline Ischemic time $\geq 240$ minutes, $n(\%)$ & $24(39.3)$ & $|5|(32.2)$ & 0.2641 \\
\hline TREs $\leq 30$ days after $\mathrm{HTX}$, mean $\pm \mathrm{SD}$ & $0.2 \pm 0.6$ & $0.3 \pm 0.6$ & 0.8979 \\
\hline$\geq \mathrm{I}$ TRE $\leq 30$ days after HTX, $\mathrm{n}(\%)$ & II (I8.0) & $92(19.6)$ & 0.7688 \\
\hline Biatrial HTX, n (\%) & $28(45.9)$ & $133(28.4)$ & $0.005 I^{*}$ \\
\hline Bicaval HTX, n (\%) & $17(27.9)$ & $98(20.9)$ & 0.2139 \\
\hline Total orthotopic HTX, n (\%) & $16(26.2)$ & $238(50.7)$ & $0.0003^{*}$ \\
\hline Length of initial hospital stay (days), mean \pm SD & $42.5 \pm 21.8$ & $43.0 \pm 22.8$ & 0.8480 \\
\hline
\end{tabular}

Notes: a GFR $<60 \mathrm{~mL} / \mathrm{min} / 1.73 \mathrm{~m}^{2} ; * \mathrm{P}<0.05$, statistically significant.

Abbreviations: HTX, heart transplantation; SD, standard deviation; GFR, glomerular filtration rate; CABG, coronary artery bypass graft; VAD, ventricular assist device; CMP, cardiomyopathy; m, male; f, female; TRE, treated rejection episode; n, number.

(end-diastolic diameter $<40 \mathrm{~mm}$ ) than those with BA-HTX $(4.3 \%)$ or BC-HTX (47.0\%). There were no statistically significant differences between HTX techniques regarding right ventricular size, left ventricular size, left ventricular ejection fraction, mitral regurgitation, or tricuspid regurgitation (all $P \geq 0.0500$ ).

\section{By heart rhythm}

Patients with AF had a higher rate of an enlarged right atrium (73.8\% vs $42.0 \%, P<0.0001)$, an enlarged left atrium (77.0\% vs $49.0 \%, P<0.0001)$, and an enlarged right ventricle (32.8\% vs $13.9 \%, P=0.0002)$. Moreover, patients with AF had significantly more often mitral regurgitation $(41.0 \%$ vs $21.5 \%, P=0.0008$ ), whereas tricuspid regurgitation was not statistically different between groups $(P=0.0880)$. No statistically significant difference was found regarding left ventricular size or left ventricular ejection fraction (both $P \geq 0.0500$ ). Echocardiographic features within 30 days after HTX are given in Table 4.

\section{Multivariate risk factor analysis for early posttransplant AF}

Multivariate risk factor analysis for posttransplant AF within 30 days after HTX included the following seven 
Table 3 Medication within 30 days after HTX

\begin{tabular}{|c|c|c|c|c|}
\hline Parameters & Biatrial $(n=161)$ & Bicaval $(n=I \mid 5)$ & Total orthotopic $(n=254)$ & $P$-value \\
\hline \multicolumn{5}{|l|}{ (A) By HTX technique } \\
\hline Cyclosporine A, n (\%) & $159(98.8)$ & $49(42.6)$ & $132(52.0)$ & $<0.0001 *$ \\
\hline Tacrolimus, n (\%) & $2(1.2)$ & $66(57.4)$ & $122(48.0)$ & $<0.000 I^{*}$ \\
\hline Azathioprine, n (\%) & $159(98.8)$ & $30(26.1)$ & $78(30.7)$ & $<0.000 I^{*}$ \\
\hline Mycophenolate mofetil, n (\%) & $2(1.2)$ & $85(73.9)$ & $176(69.3)$ & $<0.000 I^{*}$ \\
\hline Steroids, n (\%) & $161(100.0)$ & $115(100.0)$ & $254(100.0)$ & na \\
\hline ASA, n (\%) & $9(5.6)$ & $10(8.7)$ & $22(8.7)$ & 0.4743 \\
\hline Beta blocker, n (\%) & $21(13.0)$ & $21(18.3)$ & $37(14.6)$ & 0.4762 \\
\hline Ivabradine, n (\%) & $0(0.0)$ & $13(11.3)$ & $17(6.7)$ & $0.0002 *$ \\
\hline Calcium channel blocker, n (\%) & $30(18.6)$ & $25(21.7)$ & $74(29.1)$ & $0.0400 *$ \\
\hline Dihydropyridine & $8(5.0)$ & $8(6.9)$ & $35(13.8)$ & $0.0068 *$ \\
\hline Non-dihydropyridine & $22(13.7)$ & $17(14.8)$ & $39(15.4)$ & 0.8937 \\
\hline ACE inhibitor/ARB, $n(\%)$ & $70(43.5)$ & $44(38.3)$ & $126(49.6)$ & 0.1099 \\
\hline Diuretic, n (\%) & $161(100.0)$ & $115(100.0)$ & $254(100.0)$ & na \\
\hline Statin, n (\%) & $7(4.3)$ & $49(42.6)$ & $134(52.8)$ & $<0.000 I^{*}$ \\
\hline \multirow[t]{2}{*}{ Gastric protection (PPI/ $\mathrm{H}_{2}$ blocker), n (\%) } & $161(100.0)$ & $115(100.0)$ & $254(100.0)$ & na \\
\hline & \multicolumn{2}{|c|}{ Atrial fibrillation $(n=6 I)$} & Sinus rhythm $(n=469)$ & \\
\hline \multicolumn{5}{|c|}{ (B) By heart rhythm within 30 days after HTX } \\
\hline Cyclosporine A, n (\%) & \multicolumn{2}{|l|}{$37(60.7)$} & $303(64.6)$ & 0.5451 \\
\hline Tacrolimus, $\mathrm{n}(\%)$ & \multicolumn{2}{|l|}{$24(39.3)$} & $166(35.4)$ & 0.5451 \\
\hline Azathioprine, n (\%) & \multicolumn{2}{|l|}{$33(54.1)$} & $234(49.9)$ & 0.5366 \\
\hline Mycophenolate mofetil, n (\%) & \multicolumn{2}{|l|}{$28(45.9)$} & $235(50.1)$ & 0.5366 \\
\hline Steroids, n (\%) & \multicolumn{2}{|l|}{$61(100.0)$} & $469(100.0)$ & na \\
\hline ASA, n (\%) & \multicolumn{2}{|l|}{$4(6.6)$} & $37(7.9)$ & 0.7142 \\
\hline Beta blocker, n (\%) & \multicolumn{2}{|l|}{ II (I8.0) } & $68(14.5)$ & 0.4660 \\
\hline Ivabradine, n (\%) & \multicolumn{2}{|l|}{$2(3.3)$} & $28(6.0)$ & 0.3922 \\
\hline Calcium channel blocker, n (\%) & \multicolumn{2}{|l|}{$14(22.9)$} & $115(24.5)$ & 0.7882 \\
\hline Dihydropyridine & \multicolumn{2}{|l|}{$3(4.9)$} & $48(10.2)$ & 0.1853 \\
\hline Non-dihydropyridine & \multicolumn{2}{|l|}{ II (I8.0) } & $67(14.3)$ & 0.4371 \\
\hline ACE inhibitor/ARB, $n(\%)$ & \multicolumn{2}{|l|}{$21(34.4)$} & $219(46.7)$ & 0.0702 \\
\hline Diuretic, n (\%) & \multicolumn{2}{|l|}{$61(100.0)$} & $469(100.0)$ & na \\
\hline Statin, $n(\%)$ & \multicolumn{2}{|l|}{$19(31.1)$} & I7| (36.5) & 0.4157 \\
\hline Gastric protection (PPI/ $\mathrm{H}_{2}$ blocker), n (\%) & \multicolumn{2}{|l|}{$61(100.0)$} & $469(100.0)$ & na \\
\hline
\end{tabular}

Note: $* P<0.05$, statistically significant.

Abbreviations: HTX, heart transplantation; ASA, acetylsalicylic acid; ACE inhibitor, angiotensin-converting-enzyme inhibitor; ARB, angiotensin II receptor blocker; PPI, proton pump inhibitor; $\mathrm{H}_{2}$ blocker, histamine receptor blocker; $\mathrm{n}$, number; na, not applicable.

variables: valvular heart disease as principal diagnosis for HTX (hazard ratio [HR]: 2.390; 95\% confidence interval [CI]: $1.053-5.423 ; P=0.0372)$, donor age ( $\geq 40$ years; HR: 1.736, CI: 0.985-3.060, $P=0.0566$ ), prolonged ischemic time ( $\geq 240$ minutes; HR: 1.725 , CI: 0.999-2.978, $P=0.0504$ ), occurrence of a TRE within 30 days after HTX (HR: 0.759, CI: $0.388-1.484, P=0.4204)$, an enlarged end-diastolic left atrial diameter after HTX ( $\geq 40$ mm; HR: 2.498, CI: 1.291-4.835, $P=0.0066)$, mitral regurgitation after HTX (HR: 1.763 , CI: 1.035-3.004, $P=0.0370$ ), and non-TO-HTX (BA and BC; HR: 2.236, CI: $1.200-4.164, P=0.0112$ ). Data on multivariate analysis for posttransplant AF within 30 days after HTX are given in Table 5.

\section{Survival}

\section{By HTX technique}

There was no statistically significant difference in 5-year survival between patients with BA-HTX (71.2\%), BC-HTX (63.7\%), or TO-HTX (68.6\%) in the KaplanMeier survival analysis $(P=0.4458)$. A Kaplan-Meier estimator (5-year survival of patients by HTX technique) is shown in Figure 1.

\section{By heart rhythm}

Kaplan-Meier survival analysis showed a statistically significant 5-year survival $(P<0.0001)$ in patients with SR $(71.4 \%)$ compared to those with AF (44.4\%). A Kaplan-Meier 
Table 4 Echocardiographic features within 30 days after HTX

\begin{tabular}{|c|c|c|c|c|}
\hline Parameters & Biatrial $(n=161)$ & Bicaval $(n=115)$ & Total orthotopic $(n=254)$ & $P$-value \\
\hline \multicolumn{5}{|l|}{ (A) By HTX technique } \\
\hline \multicolumn{5}{|l|}{ End-diastolic diameter } \\
\hline Normal RA (<35 mm), n (\%) & $7(4.3)$ & $84(73.0)$ & $197(77.6)$ & $<0.0001 *$ \\
\hline Normal LA (<40 mm), n (\%) & $7(4.3)$ & $54(47.0)$ & $192(75.6)$ & $<0.000 I^{*}$ \\
\hline Normal RV (<30 mm), n (\%) & $140(87.0)$ & $92(80.0)$ & $213(83.9)$ & 0.2990 \\
\hline Normal LV (<55 mm), n (\%) & I45 (90.I) & $109(94.8)$ & $238(93.7)$ & 0.2464 \\
\hline \multicolumn{5}{|l|}{ LVEF } \\
\hline$\geq 55 \%, n(\%)$ & I43 (88.8) & $107(93.0)$ & $232(9 \mid .3)$ & 0.4618 \\
\hline$<55 \%, \mathrm{n}(\%)$ & $18(\mid 1.2)$ & $8(7.0)$ & $22(8.7)$ & \\
\hline $45 \%-54 \%$ & $2(1.2)$ & $3(2.6)$ & $7(2.8)$ & \\
\hline $30 \%-44 \%$ & $2(1.2)$ & $0(0.0)$ & $5(2.0)$ & \\
\hline$<30 \%$ & $14(8.8)$ & $5(4.4)$ & $10(3.9)$ & \\
\hline \multicolumn{5}{|l|}{ Mitral regurgitation } \\
\hline No, n (\%) & 119 (73.9) & $88(76.5)$ & $197(77.6)$ & 0.6942 \\
\hline Yes, n (\%) & $42(26.1)$ & $27(23.5)$ & $57(22.4)$ & \\
\hline Mild & $4 \mid(25.5)$ & $27(23.5)$ & $55(21.6)$ & \\
\hline Moderate & I (0.6) & $0(0.0)$ & $2(0.8)$ & \\
\hline Severe & $0(0.0)$ & $0(0.0)$ & $0(0.0)$ & \\
\hline \multicolumn{5}{|l|}{ Tricuspid regurgitation } \\
\hline No, $n(\%)$ & $106(65.8)$ & $64(55.7)$ & $169(66.5)$ & 0.1097 \\
\hline Yes, n (\%) & $55(34.2)$ & $5 I(44.3)$ & $85(33.5)$ & \\
\hline Mild & $31(19.2)$ & $27(23.5)$ & $54(21.3)$ & \\
\hline Moderate & $12(7.5)$ & $18(15.7)$ & $22(8.7)$ & \\
\hline \multirow[t]{2}{*}{ Severe } & $12(7.5)$ & $6(5.2)$ & $9(3.5)$ & \\
\hline & \multicolumn{2}{|c|}{ Atrial fibrillation $(n=6 I)$} & Sinus rhythm $(n=469)$ & \\
\hline \multicolumn{5}{|c|}{ (B) By heart rhythm within 30 days after HTX } \\
\hline \multicolumn{5}{|l|}{ End-diastolic diameter } \\
\hline Normal RA (<35 mm), n (\%) & $16(26.2)$ & & $272(58.0)$ & $<0.000 I^{*}$ \\
\hline Normal LA (<40 mm), n (\%) & $14(23.0)$ & & $239(51.0)$ & $<0.000 I^{*}$ \\
\hline Normal RV (<30 mm), n (\%) & $41(67.2)$ & & $404(86.1)$ & $0.0002 *$ \\
\hline Normal LV (<55 mm), n (\%) & $55(90.2)$ & & $437(93.2)$ & 0.3909 \\
\hline \multicolumn{5}{|l|}{ LVEF } \\
\hline$\geq 55 \%, \mathrm{n}(\%)$ & $52(85.2)$ & & $430(91.7)$ & 0.0993 \\
\hline$<55 \%, \mathrm{n}(\%)$ & $9(14.8)$ & & $39(8.3)$ & \\
\hline $45 \%-54 \%$ & $3(4.9)$ & & $9(1.9)$ & \\
\hline $30 \%-44 \%$ & $\mathrm{I}(\mathrm{l} .7)$ & & $6(1.3)$ & \\
\hline$<30 \%$ & $5(8.2)$ & & $24(5.1)$ & \\
\hline \multicolumn{5}{|l|}{ Mitral regurgitation } \\
\hline No, n (\%) & $36(59.0)$ & & $368(78.5)$ & $0.0008^{*}$ \\
\hline Yes, n (\%) & $25(4 I .0)$ & & I0I (2I.5) & \\
\hline Mild & $25(4 I .0)$ & & $98(20.9)$ & \\
\hline Moderate & $0(0.0)$ & & $3(0.6)$ & \\
\hline Severe & $0(0.0)$ & & $0(0.0)$ & \\
\hline \multicolumn{5}{|l|}{ Tricuspid regurgitation } \\
\hline No, n (\%) & $33(54.1)$ & & $306(65.2)$ & 0.0880 \\
\hline Yes, n (\%) & $28(45.9)$ & & $163(34.8)$ & \\
\hline Mild & II (I8.0) & & $101(21.5)$ & \\
\hline Moderate & $10(16.4)$ & & $42(9.0)$ & \\
\hline Severe & $7(11.5)$ & & $20(4.3)$ & \\
\hline
\end{tabular}

Note: $* P<0.05$, statistically significant.

Abbreviations: HTX, heart transplantation; RA, right atrium; LA, left atrium; RV, right ventricle; LV, left ventricle; LVEF, left ventricular ejection fraction; n, number. 
Table 5 Multivariate analysis for posttransplant atrial fibrillation within 30 days after HTX

\begin{tabular}{llll}
\hline Variables & Hazard ratio & 95\% Confidence interval & P-value \\
\hline Valvular heart disease as the principal diagnosis for HTX (in total) & 2.390 & $1.053-5.423$ & $0.0372^{*}$ \\
Donor age ( $\geq 40$ years) & 1.736 & $0.985-3.060$ & 0.0566 \\
Prolonged ischemic time ( $\geq 240$ minutes) & 1.725 & $0.999-2.978$ & 0.0504 \\
$\geq$ I treated rejection episode $\leq 30$ days after HTX (in total) & 0.759 & $0.388-1.484$ & 0.4204 \\
Enlarged left atrial diameter after HTX ( $\geq 40 \mathrm{~mm})$ & 2.498 & $1.291-4.835$ & $0.0066^{*}$ \\
Mitral regurgitation after HTX (in total) & 1.763 & $1.035-3.004$ & $0.0370^{*}$ \\
Non-total orthotopic HTX (in total) & 2.236 & $1.200-4.164$ & $0.0112^{*}$ \\
\hline
\end{tabular}

Note: $* P<0.05$, statistically significant.

Abbreviation: HTX, heart transplantation.

estimator (5-year survival of patients by heart rhythm) is shown in Figure 2.

Comparing 5-year follow-up mortality rates, we found that patients with AF had a higher mortality rate with BA-HTX (AF: 12 of 25 [48.0\%], SR: 34 of 132 [25.8\%], $P=0.0251$ ), with BC-HTX (AF: 12 of 14 [85.7\%], SR: 28 of 65 [43.1\%], $P=0.0038$ ), and with TO-HTX (AF: 8 of 10 [80.0\%], SR: 68 of 200 [34.0\%], $P=0.0031)$.

Furthermore, 5 -year survival analysis of patients by HTX technique and heart rhythm showed a statistically significant difference $(P<0.0001)$. Patients with SR had better survival irrespectively of the surgical technique (BA: SR [74.3\%] vs AF [56.0\%], BC: SR [70.2\%] vs AF [23.5\%], TO: SR [70.2\%] vs AF [45.8\%]). A Kaplan-Meier estimator (5-year survival of patients by HTX technique and heart rhythm) is shown in Figure 3.

\section{Discussion}

\section{Incidence of early posttransplant AF}

In this study, 61 patients (11.5\%) developed AF within 30 days after HTX. These patients were subdivided into 28 patients with BA-HTX (17.4\%), 17 patients with BC-HTX (14.8\%), and 16 patients with TO-HTX $(6.3 \% ; P=0.0012)$. This is in

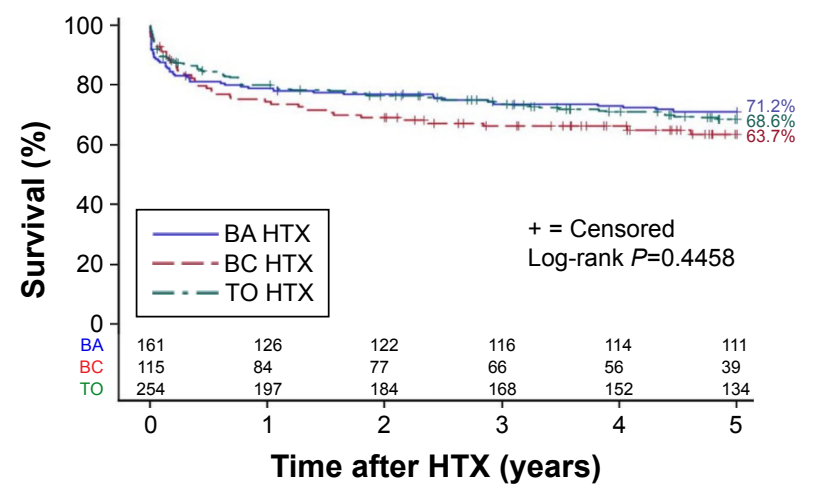

Figure I Kaplan-Meier estimator (5-year survival by surgical technique). Note: Five-year survival of patients stratified by surgical technique (BA, BC, and TO HTX).

Abbreviations: HTX, heart transplantation; BA, biatrial; BC, bicaval; TO, total orthotopic. line with the findings of Dasari et $\mathrm{al}^{1}$ who observed a 30-day incidence of AF with $7.9 \%$ after HTX and of Pavri et $\mathrm{al}^{2}$ who reported a 14-day incidence of AF with 18.2\% after HTX.

\section{Techniques of HTX}

In patients with BA-HTX, the donor atria are joined with most of the recipient atria, including pulmonary veins, superior vena cava, and inferior vena cava. As a result, both atrial cavities are enlarged resulting in distorted anatomy with potentially proarrhythmic scar tissue and previously reported atrial arrhythmias with even recipient-to-donor atrioatrial conduction. ${ }^{13,14}$ In contrast, BC-HTX and TO-HTX preserved the geometry and conduction system of the right donor atrium, as well as these keep most of the left donor atrium intact. ${ }^{4,5}$ Consequently, a lower incidence of atrial arrhythmias compared to BA-HTX has been observed. ${ }^{8}$ However, differences in size of the left atrial diameter and the remaining left atrial tissue between the two previously mentioned techniques still exist. BC-HTX features one large piece of recipient left atrium including the pulmonary vein ostia, whereas TO-HTX has two smaller pieces of recipient left atrium consisting of one atrial graft with the right pulmonary veins and another with the left pulmonary veins. ${ }^{6,7}$

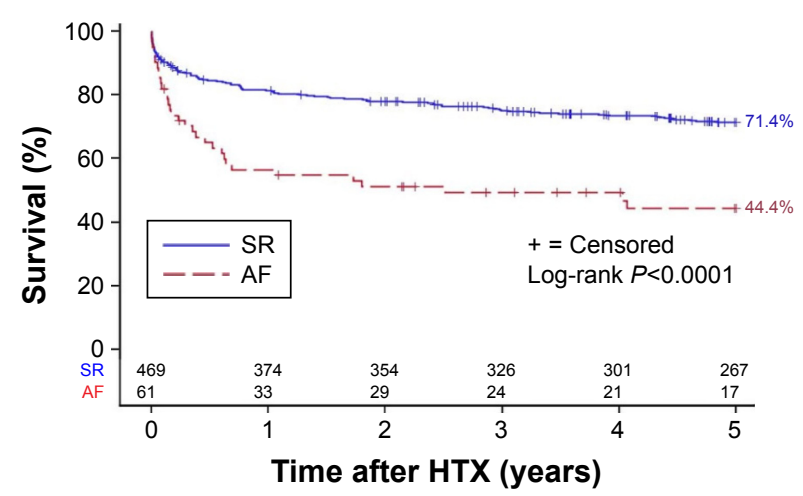

Figure 2 Kaplan-Meier estimator (5-year survival by heart rhythm). Note: Five-year survival of patients stratified by heart rhythm within 30 days after HTX (SR or AF).

Abbreviations: $\mathrm{HTX}$, heart transplantation; SR, sinus rhythm; AF, atrial fibrillation. 


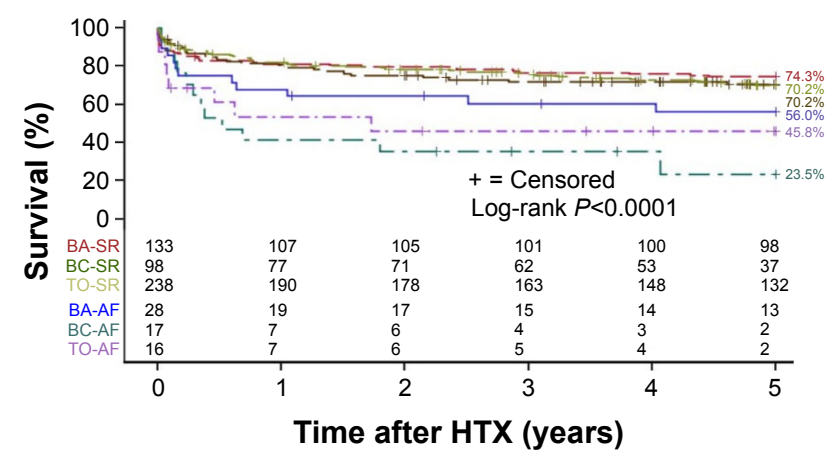

-- - Biatrial HTX, sinus rhythm (BA-SR)
- - Bicaval HTX, sinus rhythm (BC-SR)
- - Total orthotopic HTX, sinus rhythm (TO-SR)
- Biatrial HTX, atrial fibrillation (BA-AF)
-- - Bicaval HTX, atrial fibrillation (BC-AF)
$--\cdot-$ Total orthotopic HTX, atrial fibrillation (TO-AF)

Figure 3 Kaplan-Meier estimator (5-year survival by surgical technique and heart rhythm).

Note: Five-year survival of patients stratified by surgical technique (biatrial, bicaval, and total orthotopic HTX) and by heart rhythm within 30 days after HTX (SR or AF).

Abbreviation: HTX, heart transplantation.

We found the lowest rate of AF within 30 days after HTX in patients with TO-HTX $(6.3 \%)$, followed by BC-HTX (14.8\%) and BA-HTX $(17.4 \%, P=0.0012)$. These findings suggest that TO-HTX is associated with a reduced occurrence of early posttransplant AF due to maintenance of atrial anatomic integrity and physiology.

However, the selection of surgical technique depends on various factors, such as surgeon's preference, anatomical characteristics, and previous open heart surgery, and should therefore be subject to a case-by-case decision by the surgical team in the patient's best interest.

\section{Risk factor analysis for early posttransplant AF}

Several risk factors may influence the occurrence of early posttransplant AF. Prolonged ischemic time has been associated with an increased rate of posttransplant AF, especially patients with a prolonged ischemic time ( $\geq 240$ minutes) are at higher risk. ${ }^{15}$ As BA-HTX has a shorter ischemic time due to fewer anastomoses, this might be associated with a decreased rate of AF. However, we found no significant differences in ischemic time or prolonged ischemic time between patients with $\mathrm{SR}$ and $\mathrm{AF}$ in the univariate $(P=0.1618$ and $P=0.2641$, respectively) or in the multivariate analysis ( $P=0.0504)$. This is in line with previous reports' finding of no association between ischemic time and AF. ${ }^{1,2}$

Atrial tachyarrhythmias after HTX have been linked to acute rejection. ${ }^{16,17}$ We did not observe any association between acute rejection and AF. Neither did the number of TREs $(P=0.8979)$ nor the occurrence of $\geq 1$ TRE differ in the univariate $(P=0.7688)$ or multivariate analysis $(P=0.4204)$. Other studies reported similar findings as they did not observe any association between acute rejection and AF after HTX. ${ }^{1,2}$ Moreover, we did not find any significant differences in the immunosuppressive drug therapy (CsA, TAC, AZA, or MMF) between patients with or without AF.

Antiarrhythmic drugs (beta blocker or calcium channel blocker) can influence the occurrence of AF. ${ }^{11}$ Comparing the initial medication within 30 days after HTX, we found no significant differences between patients with SR or AF with regard to the use of beta blocker or calcium channel blocker. In a previous study, our group analyzed the influence of the long-term use of amiodarone before HTX on early posttransplant AF and could demonstrate that long-term use of amiodarone prior to HTX significantly reduced early posttransplant AF. ${ }^{11}$ A comparison of the three HTX techniques regarding pretransplant long-term use of amiodarone showed no significant difference $(P=0.3042)$.

Univariate and multivariate analyses of echocardiographic features showed that patients with AF had a higher rate of an enlarged left atrium $(P<0.0001$ and $P=0.0066$, respectively). Patients with a dilated left atrium may be more susceptible to AF due to increased wall tension and excitable scar tissue. Patients with BA-HTX and BC-HTX had higher rates of an enlarged left atrium compared to those with TO-HTX $(P<0.0001)$. Former studies have stated worse outcomes with BA-HTX regarding atrial diameter, atrial geometry, and atrial hemodynamics resulting in an increased rate of $\mathrm{AF}^{4,5,9}$ whereas TO-HTX offers a more physiologic atrial anatomy and size with improved left atrial function. ${ }^{18}$

We observed a higher number of mitral regurgitation in patients with $\mathrm{AF}$ in the univariate and multivariate analyses ( $P=0.0008$ and $P=0.0370$, respectively), although there were mainly mild forms of mitral regurgitation. However, even mild forms of mitral regurgitation may provoke atrial arrhythmias, such as AF, by jet-induced stretching of the atrium or provoking rapidly discharging triggers, especially in cases of an already enlarged left atrium. Various studies have reported an increased incidence of posttransplant mitral regurgitation in patients with BA-HTX. ${ }^{4,5,18}$ Moreover, mitral regurgitation may be related to abnormal atrial size causing impaired valvular integrity and function. ${ }^{19}$ We detected the highest rate of mitral regurgitation in patients with BA-HTX (26.1\%), followed by BC-HTX (23.5\%) and TO-HTX (22.4\%).

Increase of the left atrial diameter and mitral regurgitation after HTX as risk factors for AF highlight the importance of 
preserved anatomic integrity. As a matter of fact, TO-HTX itself significantly reduced early posttransplant AF by creating normal sized atrial diameters along with a lower rate of mitral regurgitation.

Patients with valvular heart disease as principal diagnosis for HTX had a higher rate of early posttransplant AF which might be due to former open heart surgery causing scar tissue and adhesions. This could result in aggravated anastomoses of the donor heart providing potential foci for arrhythmias.

\section{Survival}

The development of posttransplant AF has been associated with increased mortality after HTX. ${ }^{1,2}$ Even short periods of AF have been linked to worse outcomes. ${ }^{2}$ These reports are in line with our findings showing an inferior 5-year survival of patients with AF $(P<0.0001)$. Furthermore, our data showed an impaired survival of patients with $\mathrm{AF}$ in all three techniques.

A survival advantage in patients with BC-HTX or TO-HTX compared to BA-HTX has been controversially discussed in the literature..$^{5,8,9,20,21}$ Some studies reported a higher mortality rate with BA-HTX, ${ }^{8,9}$ whereas other publications found no difference. $5,20,21$

As a matter of fact, we could not find any statistically significant difference in 5-year survival between HTX techniques $(P=0.4458)$, although the occurrence of early posttransplant AF was higher in patients with BA-HTX and BC-HTX.

This circumstance indicates that survival after HTX depends on various risk factors of which early posttransplant AF seems to be an important one - but not the only one. Hence, this large study of 530 patients was able to demonstrate differences between the three HTX techniques regarding the occurrence of AF as well as to show the influence of early posttransplant AF on survival after HTX, in general - but not to distinguish the effect of AF on survival within the three different HTX techniques as several risk factors can affect survival after HTX. Therefore, further large prospective randomized controlled studies are required to address this interesting issue.

\section{Study limitations}

First, these findings were based on a single-center study with 530 patients. However, due to its participant number including 161 patients with BA-HTX, 115 patients with BC-HTX, and 254 patients with TO-HTX, these results may be compared in numbers with those of multicenter studies. Additionally, patients received a comparable center-specific treatment and follow-up minimizing possible confounders.
Second, although data were gathered prospectively, the retrospective nature of this analysis carries the limitations of such a study design. ${ }^{10,11}$

Third, a possible era effect cannot be ruled out, given the long follow-up period..$^{22,23}$

\section{Conclusion}

Early posttransplant AF was associated with a significantly increased mortality rate after HTX $(P<0.0001)$. A multivariate risk factor analysis indicated pretransplant valvular heart disease, an enlarged left atrium after HTX, mitral regurgitation after HTX, and non-TO (BA and BC) as significant risk factors for early posttransplant AF. Patients with TO-HTX had the lowest rate of AF within 30 days after HTX.

\section{Acknowledgments}

The authors thank Gerda Baumann, Viola Deneke, and Berthold Klein for their assistance and advice.

\section{Disclosure}

The authors report no conflicts of interest in this work.

\section{References}

1. Dasari TW, Pavlovic-Surjancev B, Patel N, et al. Incidence, risk factors, and clinical outcomes of atrial fibrillation and atrial flutter after heart transplantation. Am J Cardiol. 2010;106(5):737-741.

2. Pavri BB, O'Nunain SS, Newell JB, Ruskin JN, William G. Prevalence and prognostic significance of atrial arrhythmias after orthotopic cardiac transplantation. J Am Coll Cardiol. 1995;25(7):1673-1680.

3. Shumway NE, Lower RR, Stofer RC. Transplantation of the heart. Adv Surg. 1966;2:265-284.

4. Morgan JA, Edwards NM. Orthotopic cardiac transplantation: comparison of outcome using biatrial, bicaval, and total techniques. J Card Surg. 2005;20(1):102-106.

5. Dell'Aquila AM, Mastrobuoni S, Bastarrika G, et al. Bicaval versus standard technique in orthotopic heart transplant: assessment of atrial performance at magnetic resonance and transthoracic echocardiography. Interact Cardiovasc Thorac Surg. 2012;14(4):457-462.

6. Sievers HH, Weyand M, Kraatz EG, Bernhard A. An alternative technique for orthotopic cardiac transplantation, with preservation of the normal anatomy of the right atrium. Thorac Cardiovasc Surg. 1991;39(2): $70-72$.

7. Dreyfus G, Jebara V, Mihaileanu S, Carpentier AF. Total orthotopic heart transplantation: an alternative to the standard technique. Ann Thorac Surg. 1991;52(5):1181-1184.

8. Locali RF, Matsuoka PK, Cherbo T, Gabriel EA, Buffolo E. [Should biatrial heart transplantation still be performed?: a meta-analysis]. Arq Bras Cardiol. 2010;94(6):829-840. Portuguese [with English abstract].

9. Schnoor M, Schäfer T, Lühmann D, Sievers HH. Bicaval versus standard technique in orthotopic heart transplantation: a systematic review and meta-analysis. J Thorac Cardiovasc Surg. 2007;134(5):1322-1331.

10. Rivinius R, Helmschrott M, Ruhparwar A, et al. Analysis of malignancies in patients after heart transplantation with subsequent immunosuppressive therapy. Drug Des Devel Ther. 2014;9:93-102.

11. Rivinius R, Helmschrott M, Ruhparwar A, et al. Long-term use of amiodarone before heart transplantation reduces significantly early posttransplant atrial fibrillation and is not associated with increased mortality after heart transplantation. Drug Des Devel Ther. 2016;10:677-686. 
12. Stewart S, Winters GL, Fishbein MC, et al. Revision of the 1990 working formulation for the standardization of nomenclature in the diagnosis of heart rejection. J Heart Lung Transplant. 2005;24(11):1710-1720.

13. Strohmer B, Chen PS, Hwang C. Radiofrequency ablation of focal atrial tachycardia and atrioatrial conduction from recipient to donor after orthotopic heart transplantation. J Cardiovasc Electrophysiol. 2000; 11(10):1165-1169.

14. Lefroy DC, Fang JC, Stevenson LW, Hartley LH, Friedman PL, Stevenson WG. Recipient-to-donor atrioatrial conduction after orthotopic heart transplantation: surface electrocardiographic features and estimated prevalence. Am J Cardiol. 1998;82(4):444-450.

15. Thajudeen A, Stecker EC, Shehata M, et al. Arrhythmias after heart transplantation: mechanisms and management. J Am Heart Assoc. 2012; 1(2):e001461.

16. Scott CD, Dark JH, McComb JM. Arrhythmias after cardiac transplantation. Am J Cardiol. 1992;70(11):1061-1063.

17. Cohn WE, Gregoric ID, Radovancevic B, Wolf RK, Frazier OH. Atrial fibrillation after cardiac transplantation: experience in 498 consecutive cases. Ann Thorac Surg. 2008;85(1):56-58.

18. Freimark D, Silverman JM, Aleksic I, et al. Atrial emptying with orthotopic heart transplantation using bicaval and pulmonary venous anastomoses: a magnetic resonance imaging study. J Am Coll Cardiol. 1995; 25(4):932-936.
19. Angermann CE, Spes CH, Tammen A, et al. Anatomic characteristics and valvular function of the transplanted heart: transthoracic versus transesophageal echocardiographic findings. $J$ Heart Transplant. 1990;9(4):331-338.

20. Weiss ES, Nwakanma LU, Russell SB, Conte JV, Shah AS. Outcomes in bicaval versus biatrial techniques in heart transplantation: an analysis of the UNOS database. J Heart Lung Transplant. 2008;27(2):178-183.

21. Grande AM, Rinaldi M, D'Armini AM, et al. Orthotopic heart transplantation: standard versus bicaval technique. Am J Cardiol. 2000;85(11): 1329-1333.

22. Helmschrott M, Beckendorf J, Akyol C, et al. Superior rejection profile during the first 24 months after heart transplantation under tacrolimus as baseline immunosuppressive regimen. Drug Des Devel Ther. 2014;8: 1307-1314.

23. Helmschrott M, Rivinius R, Ruhparwar A, et al. Advantageous effects of immunosuppression with tacrolimus in comparison with cyclosporine A regarding renal function in patients after heart transplantation. Drug Des Devel Ther. 2015;9:1217-1224.
Therapeutics and Clinical Risk Management

\section{Publish your work in this journal}

Therapeutics and Clinical Risk Management is an international, peerreviewed journal of clinical therapeutics and risk management, focusing on concise rapid reporting of clinical studies in all therapeutic areas outcomes, safety, and programs for the effective, safe, and sustained use of medicines. This journal is indexed on PubMed Central, CAS,

\section{Dovepress}

EMBase, Scopus and the Elsevier Bibliographic databases. The manuscript management system is completely online and includes a very quick and fair peer-review system, which is all easy to use. Visit http://www.dovepress.com/testimonials.php to read real quotes from published authors.

Submit your manuscript here: http://www.dovepress.com/therapeutics-and-clinical-risk-management-journal 